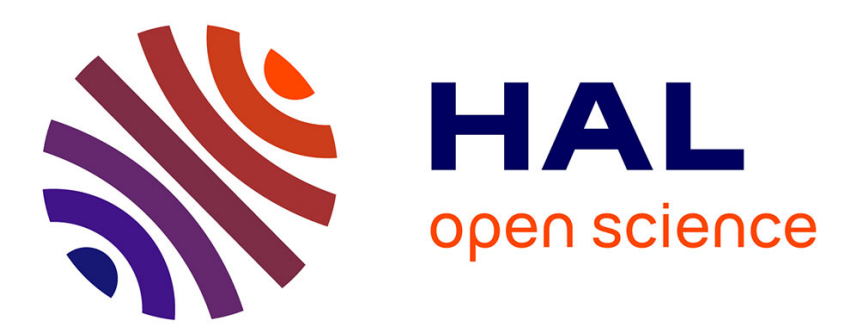

\title{
A Novel Characterization Method for Accurate Lumped Parameter Modeling of Electret Electrostatic Vibration Energy Harvesters
}

\author{
Armine Karami, Dimitri Galayko, Philippe Basset
}

\section{- To cite this version: \\ Armine Karami, Dimitri Galayko, Philippe Basset. A Novel Characterization Method for Accurate Lumped Parameter Modeling of Electret Electrostatic Vibration Energy Harvesters. IEEE Electron Device Letters, 2017, 38 (5), pp.665-668. 10.1109/LED.2017.2682232 . hal-01490740}

\author{
HAL Id: hal-01490740 \\ https://hal.science/hal-01490740
}

Submitted on 15 Mar 2017

HAL is a multi-disciplinary open access archive for the deposit and dissemination of scientific research documents, whether they are published or not. The documents may come from teaching and research institutions in France or abroad, or from public or private research centers.
L'archive ouverte pluridisciplinaire HAL, est destinée au dépôt et à la diffusion de documents scientifiques de niveau recherche, publiés ou non, émanant des établissements d'enseignement et de recherche français ou étrangers, des laboratoires publics ou privés. 


\title{
A Novel Characterization Method for Accurate Lumped Parameter Modeling of Electret Electrostatic Vibration Energy Harvesters
}

\author{
Armine Karami, Student Member, IEEE, Dimitri Galayko, Member, IEEE, and Philippe Basset
}

\begin{abstract}
This letter presents a new method for the characterization of electret transducers, which are typically used in electrostatic vibration energy harvesters (electret e-VEHs). This is the first method allowing to accurately measure the value of the equivalent voltage source representing the electret in lumped parameter models of a wide range of electret e-VEHs. An accurate value for this parameter is critical for design, analysis and optimization, given the increasing complexity of e-VEHs electrical interfaces. Until now, there was no universal method allowing the measurement of this parameter, because of practical difficulties with some geometries, and because of charging nonuniformities. In this letter, the new method is presented, with insights on how to maximize the measurement accuracy. It is then applied to a state-of-the art MEMS electret e-VEH.
\end{abstract}

Index Terms-electrostatic vibration energy harvesting, electret, charge pumps, energy conversion

\section{INTRODUCTION}

$\mathbf{E}$ LECTRET electrostatic vibration energy harvesters (eVEHs) are a promising technology to power autonomous systems [1], [2]. They are composed of a mobile mass, attached to an electret-charged transducer. The biasing of this transducer is determined by a conditioning circuit. Energy conversion occurs when the bias across the transducer results in an electrostatic force opposing the mass motion induced by an external acceleration.

In the recent years, the complexity of the available conditioning circuits has increased to optimize the harvesters performances [3]-[6]. As a result, the use of lumped parameter models of e-VEHs is becoming the sole convenient way to predict their dynamics under variable input conditions.

A convenient representation of the electret-charged transducer in lumped parameter models of e-VEHs is an equivalent constant voltage source in series with the transducer's variable capacitance [7]. This source will be referred to as the electret potential in the rest of the paper. Lumped-parameter models are expected to yield accurate predictions on the e-VEH dynamics, and to guide optimization choices in the conditioning circuit design. To do this, these models have to be parametrized accurately. In particular, the electret potential value has a critical effect on the models' predictions (chapter 11 of [8])

The electret potential is difficult to define precisely at the fabrication stage. Plus, it may decrease in large time scales [9]. Hence, it is necessary to measure its value after fabrication.

A. Karami and D. Galayko are with Sorbonne Universités, UPMC Univ Paris 06, UMR 7606, LIP6, F-75005, Paris, France (e-mail: armine.karami@lip6.fr)

P. Basset is with Université Paris-Est, ESYCOM, ESIEE Paris, France

Manuscript received March 11th, 2017.

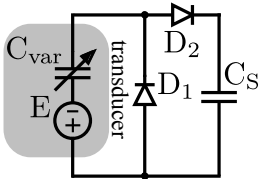

(a)

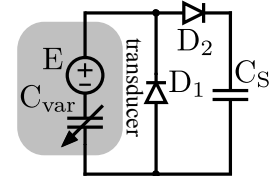

(b)
Fig. 1: Schematics of circuit HW1 (a) and circuit HW2 (b)

Until now, this was done by measuring the device's surface potential, with a contact-less electrostatic voltmeter. Ideally, this measurement has to be done between the facing capacitor plates of the transducer. However, this is impossible to perform nondestructively on a wide range of e-VEHs because of the geometry of their transducer (e.g., interdigitated-combs [10]). More importantly, this technique gives a measure of a potential which is hard to accurately relate to the value of the electret potential. In particular, its accuracy relies on the assumption of uniform charge distribution across the surfaces of the transducer's facing capacitor plates. This hypothesis is not always verified, depending on both the employed charging techniques and the transducer geometry [10], [11].

In this letter, for the first time, a non-destructive and accurate method is proposed to measure the electret potential of a given transducer. This method is compatible with a wide range of transducer geometries and charging techniques. The method is presented in Section II, with insights on how to minimize the error on the measurement. It is then applied to a state-of-the-art MEMS electret e-VEH in Section III.

\section{Presentation of the method}

\section{A. Dynamics of the measurement circuits}

The measurement method presented in this letter is based on the dynamics of the half-wave circuits HW1 and HW2, depicted in Fig. 1a and 1b, respectively. In this subsection, the dynamics of these circuits are linked to the electret potential, denoted by $E$ in the rest of the paper. The value of the capacitance at the transducer's terminals is denoted by $C_{v a r}$.

Consider the transducer as a part of the circuit configurations $\mathrm{HW} 1$ or $\mathrm{HW} 2$, with $C_{\text {var }}$ cyclically time-varying between maximum and minimum values $\left(C_{\max }, C_{\min }\right)$, subsequently to a mechanical input excitation of the e-VEH (the same input for both circuit configurations). A cycle of variation of $C_{v a r}$ is defined as its variation from $C_{\max }$ to $C_{\min }$ to $C_{\max }$. The choice of the input is not constrained as long as it results in the same values $C_{\max }$ and $C_{\min }$ at each cycle. A harmonic input is used for simplicity. In the following, $V_{1}$ 

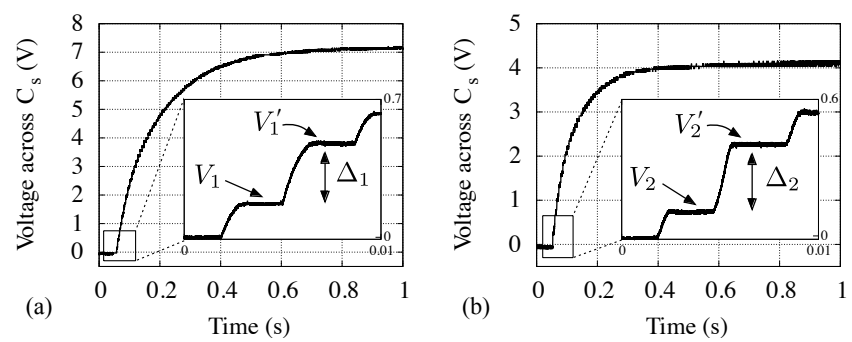

Fig. 2: Experimental measurement of $\left(\Delta_{1}, \Delta_{2}, V_{1}, V_{2}\right)$, measured on the MEMS electret transducer characterized in section III and reported in [10].

(a) Measured voltage across $C_{S}$ with HW1 circuit.

(b) Measured voltage across $C_{S}$ with HW2 circuit.

and $V_{2}$ denote the voltage across the fixed capacitor $C_{S}$ at $C_{v a r}=C_{\max }$ of a given variation cycle of $C_{v a r}$, for the HW1 and HW2 circuits respectively. The labels $V_{1}^{\prime}$ and $V_{2}^{\prime}$ denote the voltage across the fixed capacitor $C_{S}$ at $C_{v a r}=C_{\max }$ of the following cycle, for the HW1 and HW2 circuits respectively. We also define $\Delta_{1}:=V_{1}^{\prime}-V_{1}$ and $\Delta_{2}:=V_{2}^{\prime}-V_{2}$. The quantities $\left(\Delta_{1}, V_{1}\right)$ and $\left(\Delta_{2}, V_{2}\right)$ are measured on the timeevolution of the voltages across $C_{S}$ with circuits HW1 and HW2, respectively. An experimental example of measurement of $\left(\Delta_{1}, \Delta_{2}, V_{1}, V_{2}\right)$ is shown in Fig. 2.

In the aforementioned input conditions, the electrical analysis of each circuit yields the cycle-to-cycle evolution law of the voltage across $C_{S}$, which reads:

$$
\begin{aligned}
V_{1}^{\prime} & =\frac{C_{S} V_{1}+\left(C_{\max }-C_{\min }\right) E-\left(C_{\max }+C_{\min }\right) V_{T}}{C_{\min }+C_{S}} \\
V_{2}^{\prime} & =\frac{C_{S} V_{2}+\left(C_{\max }-C_{\min }\right) E-\left(C_{\max }+C_{\min }\right) V_{T}}{C_{\max }+C_{S}}
\end{aligned}
$$

where $V_{T} \geqslant 0$ denotes the fixed threshold voltage of the diodes, which are assumed to follow the ideal voltage-current diode law. From these two equations, it comes that the measured $\left(\Delta_{1}, \Delta_{2}, V_{1}, V_{2}\right)$ can be linked to $E$ and $C_{\max }$ by:

$$
\begin{aligned}
& C_{\max }=\frac{C_{S}\left(\Delta_{1}-\Delta_{2}\right)+C_{\min }\left(\Delta_{1}+V_{1}\right)}{\Delta_{2}+V_{2}}, \\
& E=\frac{\left(C_{\min }+C_{S}\right) \Delta_{1}+C_{\min } V_{1}+\left(C_{\max }+C_{\min }\right) V_{T}}{C_{\max }-C_{\min }} .
\end{aligned}
$$

The value of $C_{\min }$ can be measured by a synchronous detection method [12]. Note that in the case of interdigitatedcombs transducer geometries, $C_{\min }$ is fixed independently of the harmonic input acceleration. Hence, it can be measured by traditional means of measurement of fixed capacitances.

\section{B. Measurement errors and choice of the circuit's parameters}

To measure $E$ for a given transducer using (4), it is necessary to choose $C_{S}, V_{1}, V_{2}$, and the input acceleration. This subsection shows how these parameters have to be chosen in order to maximize the measurement accuracy.

In the following, $\delta_{V}$ denotes the uncertainty on the measured voltage across $C_{S}$ due to the superposition of random error sources in a broad sense (e.g., thermal, reading amplifier, and quantization noises). Suppose that the uncertainty on $V_{T}$ and $C_{\min }$ can be neglected, and that $V_{1}$ and $V_{2}$ are chosen to be approximatively equal to zero. The measurement uncertainties on $E$ and $C_{\max }$ due to random errors are denoted by $\delta C_{\max }$ and $\delta E$, respectively. They are linked to $\delta_{V}$ following:

$$
\begin{aligned}
\frac{\delta_{C_{\max }}}{C_{\max }} & =\delta_{V} \frac{\eta+\xi}{E \eta(\eta-1)} \sqrt{(1+\xi)^{2}+(\eta+\xi)^{2}} \\
\frac{\delta_{E}}{E} & =\delta_{V} \frac{\sqrt{(\eta+\xi)^{4}+(1+\xi)^{4}}}{E(\eta-1)^{2}}
\end{aligned}
$$

where $\xi:=C_{S} / C_{\min }$ and $\eta:=C_{\max } / C_{\min }$.

From (5-6), it comes that the measurement uncertainty due to random error sources decreases by decreasing $C_{S}$. However, decreasing $C_{S}$ increases the electromechanical coupling effect on the e-VEH's dynamics, thereby inducing a systematic measurement error on $C_{\max }$ and $E$. This is because equations (3-4) are derived from (1-2) assuming that $\left(C_{\max }, C_{\min }\right)$ with circuit HW1 (in (1)) are equal to $\left(C_{\max }, C_{\min }\right)$ with circuit HW2 (in (2)). But these circuits do not bias the transducer in the same way, and different biasing results in different electrostatic forces generated by each of the two circuits on the e-VEH's mobile mass. This, in turn, yields different mobile mass motions with each of the two circuits. Thus, in general, $\left(C_{\max }, C_{\min }\right)$ with circuit $\mathrm{HW} 1$ cannot be considered equal to $\left(C_{\max }, C_{\min }\right)$ with circuit HW2.

From the topologies of the circuits depicted in Fig. 1, it can be seen that the circuit HW1 biases the transducer with a voltage varying from $E$ at $C_{v a r}=C_{\max }$ to $E+V_{1}^{\prime}$ at $C_{v a r}=C_{\text {min }}$. The circuit HW2 biases the transducer with a voltage varying from $E$ at $C_{v a r}=C_{\min }$ to $E-V_{2}^{\prime}$ at $C_{\text {var }}=C_{\text {max }}$. By choosing $C_{S}$ large and $V_{1}, V_{2}$ as small as possible, $V_{1}^{\prime}, V_{2}^{\prime}$ are small (see (1-2)). This ensures that the biasing of $C_{v a r}$ throughout its variation is approximatively the same with both circuits (close to the voltage $E$ ). Therefore, increasing $C_{S}$ reduces the systematic measurement error due to the differential impact of the electromechanical coupling.

The following procedure is proposed to choose $C_{S}$ so as to reduce the measurement uncertainty due to random error sources, whilst ensuring that the systematic error due to the electromechanical coupling remains negligible. An admissible value for $\delta_{E} / E$ is decided a priori, and solving (6) for $\xi$ gives the corresponding $C_{S}$. Note that this supposes an a priori estimation of $\eta$ and $E$. The former can be measured by synchronous detection [12]. The latter can be estimated by one or several first inaccurate measurements (e.g., using an arbitrary value for $C_{S}$ for the first measurement). The measurements are then carried out with this value of $C_{S}$. If $C_{\max }$ obtained from (3) is consistent with its value measured by synchronous detection, then $E$ obtained from (4) using the same measured $\left(\Delta_{1}, \Delta_{2}, V_{1}, V_{2}\right)$ can be considered accurate. Otherwise, $C_{S}$ that satisfies the desired $\delta_{E} / E$ margin results in a large systematic error on $C_{\max }$, and hence on $E$. In this case, the measurement has to be carried out again with a larger $C_{S}$. The systematic error due to the electromechanical coupling will decrease, but the admissible uncertainties on $E$ and $C_{\max }$ due to random error sources will increase.

Finally, the measurement uncertainty can also be decreased by increasing $\eta$ at fixed $C_{S}$. This is done by increasing the amplitude of the mechanical harmonic input. But as $\eta$ results 


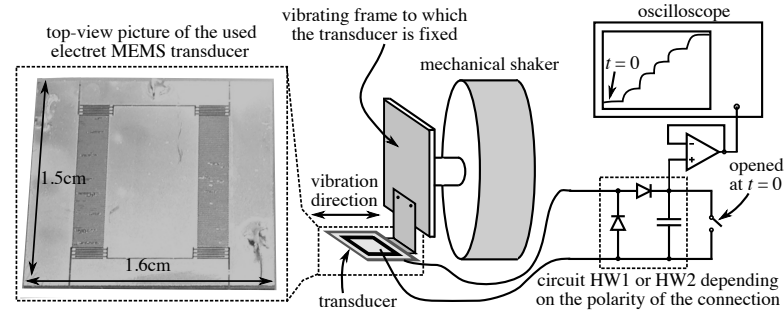

Fig. 3: Setup used for the measurement of the electret potential, with a top-view picture of the electret MEMS transducer. The device dimensions are $1.5 \mathrm{~cm} \times 1.6 \mathrm{~cm} \times 380 \mu \mathrm{m}$.

from the dynamics of the e-VEH's mechanical part, it is harder to use the input amplitude as a control parameter on the error.

Note that a technique employing the HW1 circuit has been used for electret potential measurement in [10], similar to the method presented in this paper, but using saturation voltages instead of local evolution voltages. Because of this, the method in [10] only holds if both $C_{\max }$ and $C_{\min }$ are fixed throughout the voltage evolution until saturation. This may not be verified because of the electromechanical coupling.

\section{EXPERIMENTAL RESULTS}

In this section, the method is applied to a micro-scale electrostatic transducer realized in MEMS technology, similar to the device reported in [10]. As the transducer has an interdigitated-combs geometry, $C_{\min }$ is fixed, and $C_{\max }$ alternates between two different values at each period of the harmonic mechanical input excitation [10]. Therefore, there exists two values of $C_{\max }$ for each harmonic input that the device is submitted to. Two input are chosen: $1.5 \mathrm{~g}$ and $2 \mathrm{~g}$ amplitude, both of $150 \mathrm{~Hz}$ frequency.

In the setup used for this letter, the device is continuously submitted to the input vibrations, and $C_{S}$ is first short circuited. Then, the short circuit is opened and the voltage evolution across $C_{S}$ starts. But the time at which the short circuit is opened cannot be exactly chosen as corresponding to an extrema of $C_{v a r}$. To ensure that the captured evolution results from a complete cycle of variation of $C_{v a r}$, the first cycle of voltage evolution across $C_{S}$ is ignored with both circuits. Hence, $V_{1}, V_{2}$ are slightly above zero (see Fig. 2).

To estimate the influence of random error sources on the measurement using (5-6), $E$ is first estimated at $20 \mathrm{~V}$, by a surface potential measurement done $1 \mathrm{~mm}$ on top of the device using a contact-less electrostatic voltmeter. The four values of $\eta$ are measured by synchronous detection, and $\delta_{V}$ is measured as $25 \mathrm{mV}$. Using (6), the theoretical uncertainty on $E$ decreases from $16.6 \%$ with the smallest $C_{\max }$, to $4.2 \%$ with the largest $C_{\max }$, all with $C_{S}=100 \mathrm{pF}$.

These uncertainty intervals are satisfactory for this example, so the fixed capacitor is chosen as $C_{S}=100 \mathrm{pF}$. Using an impedance-meter, $C_{\min }$ is measured as $C_{\min }=60 \mathrm{pF}$. The used diodes are JPAD5, whose parasitic capacitances values are negligible compared to $C_{S}$, and $V_{T}$ is measured as $V_{T}=0.7 \mathrm{~V}$ for typical values of current in our application.

The results of the measurement method are depicted in Fig. 4. Each point corresponds to $\left(E, C_{\max }\right)$ obtained by averaging 5 measurements of $\left(\Delta_{1}, \Delta_{2}, V_{1}, V_{2}\right)$. The error bars

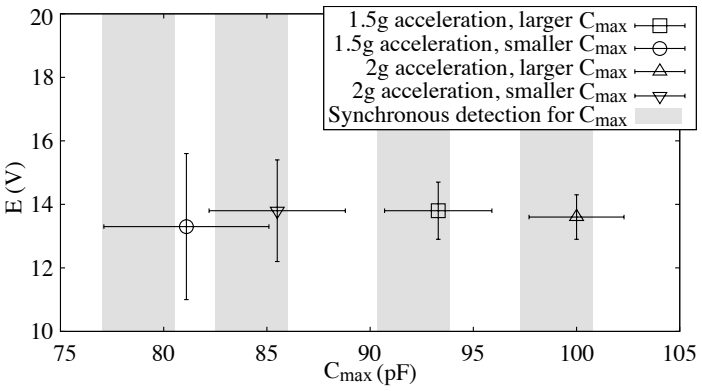

Fig. 4: Measurement results for $E$ and $C_{\max }$.

represent the uncertainties obtained on $\left(\Delta_{1}, \Delta_{2}, V_{1}, V_{2}\right)$ propagated to $\left(E, C_{\max }\right)$. An example of a single measurement of $\left(\Delta_{1}, \Delta_{2}, V_{1}, V_{2}\right)$ obtained with the present device is depicted in Fig. 2. The results in Fig. 4 show that the obtained $C_{\max }$ are in accordance with those measured by synchronous detection. This guarantees that the systematic error due to the electromechanical coupling is negligible. As expected, the uncertainty on $E$ decreases when the input amplitude increases (see end of Section II-B). Using the most accurate result, the electret potential is measured as $E=13.7 \mathrm{~V} \pm 5.1 \%$. The uncertainty is slightly increased compared to the theoretical uncertainty obtained from (6), because of the lower effective value of $E$ compared to its first estimation, and $V_{1}, V_{2}>0$.

For further validation, a voltage source of $5 \mathrm{~V}$ is added in series with the transducer to simulate a different value of the electret potential, and the measurement is carried out again. The expected electret potential is then of $E+5$ by superposition. The used input is of $2 \mathrm{~g}$ amplitude and $150 \mathrm{~Hz}$ frequency. The synchronous detection method gives $C_{\max }=102 \mathrm{pF} \pm 3 \%$ (the lower value of $C_{\max }$ is discarded). From (6), keeping $C_{S}=100 \mathrm{pF}$ results in an error of $3.3 \%$ on $E$. Using this value of $C_{S}$, the measurement yields a value of $E=23.1 \mathrm{~V} \pm 3.8 \%$ and $C_{\max }=93 \mathrm{pF} \pm 3.1 \%$. The value of $C_{\max }$ does not agree with what is measured by synchronous detection, which means that the systematic error due to the electromechanical coupling is large. Hence, the error minimization procedure discussed at the end of section II-B is applied: the admissible random error on $E$ is relaxed to $7.5 \%$, yielding $C_{S}=180 \mathrm{pF}$. The new measurement gives $C_{\max }=103 \mathrm{pF} \pm 4.2 \%$, which is in accordance with the value measured by synchronous detection. The electret potential is measured as $E=19 \mathrm{~V} \pm 6.8 \%$, which is the expected value after adding the $5 \mathrm{~V}$ external voltage.

\section{CONCLUSION}

This letter presented a method to accurately measure the value of the voltage source representing the electret in lumped parameter models of e-VEHs. The method was illustrated on an interdigitated-combs MEMS transducer, for which a traditional surface potential measurement across the transducer is hard to carry out and gives inaccurate results. The results were verified by comparing the maximum capacitance value, which is obtained as an intermediary parameter in the reported method, with its value measured by synchronous detection. The method was also validated by changing the charging conditions and checking the coherence of the results. 


\section{REFERENCES}

[1] P. Pondrom, G. Sessler, J. Bös, and T. Melz, "Compact electret energy harvester with high power output," Applied Physics Letters, vol. 109, no. 5, p. 053906, August 2016. doi: 10.1063/1.4960480

[2] Y. Suzuki, "Electret based vibration energy harvester for sensor network," in 2015 Transducers: The 18th International Conference on Solid-State Sensors, Actuators and Microsystems. IEEE, June 2015. doi: 10.1109/TRANSDUCERS.2015.7180856 pp. 43-46.

[3] D. Miki, Y. Suzuki, and N. Kasagi, "Effect of nonlinear external circuit on electrostatic damping force of micro electret generator," in 2009 Transducers: The 15th International Conference on Solid-State Sensors, Actuators and Microsystems. IEEE, June 2009. doi: 10.1109/SENSOR.2009.5285405 pp. 636-639.

[4] S. Boisseau, P. Gasnier, M. Gallardo, and G. Despesse, "Self-starting power management circuits for piezoelectric and electret-based electrostatic mechanical energy harvesters," in Journal of Physics: Conference Series, vol. 476, no. 1. IOP Publishing, 2013. doi: 10.1088/17426596/476/1/012080 p. 012080 .

[5] J. Wei, E. Lefeuvre, H. Mathias, and F. Costa, "Interface circuit with adjustable bias voltage enabling maximum power point tracking of capacitive energy harvesting devices," Journal of Micromechanics and Microengineering, vol. 26, no. 12, p. 124008, 2016. doi: 10.1088/0960$1317 / 26 / 12 / 124008$

[6] A. Karami, D. Galayko, and P. Basset, "Series-parallel charge pump conditioning circuits for electrostatic kinetic energy harvesting," IEEE Transactions on Circuits and Systems I: Regular Papers, vol. 64, no. 1, pp. 227-240, Jan 2017. doi: 10.1109/TCSI.2016.2603064

[7] S. Boisseau, G. Despesse, and B. A. Seddik, "Electrostatic conversion for vibration energy harvesting," in Small-Scale Energy Harvesting. Intech, 2012, pp. 91-134. ISBN 978-953-51-0826-9

[8] P. Basset, E. Blokhina, and D. Galayko, Electrostatic Kinetic Energy Harvesting. John Wiley \& Sons, 2016. ISBN 978-1-84821-716-4

[9] S. Boisseau, J. Chaillout, J. Danel, J. Legras, and G. Despesse, "Stable drie-patterned sio 2/si 3 n 4 electrets," in 2013 Transducers Eurosensors XXVII: The 17th International Conference on Solid-State Sensors, Actuators and Microsystems. IEEE, 2013. doi: 10.1109/Transducers.2013.6627174 pp. 1942-1945.

[10] Y. Lu, E. ORiordan, F. Cottone, S. Boisseau, D. Galayko, E. Blokhina, F. Marty, and P. Basset, "A batch-fabricated electret-biased wideband mems vibration energy harvester with frequency-up conversion behavior powering a uhf wireless sensor node," Journal of Micromechanics and Microengineering, vol. 26, no. 12, p. 124004, September 2016. doi: 10.1088/0960-1317/26/12/124004

[11] M. Renaud, G. Altena, R. Elfrink, M. Goedbloed, C. de Nooijer, and R. van Schaijk, "Modeling and characterization of electret based vibration energy harvesters in slot-effect configuration," Smart Materials and Structures, vol. 24, no. 8, p. 085023, July 2015. doi: 10.1088/0964$1726 / 24 / 8 / 085023$

[12] P. Basset, D. Galayko, A. M. Paracha, F. Marty, A. Dudka, and T. Bourouina, "A batch-fabricated and electret-free silicon electrostatic vibration energy harvester," Journal of Micromechanics and Microengineering, vol. 19 , no. 11, p. 115025 , October 2009. doi: 10.1088/0960$1317 / 19 / 11 / 115025$ 